\title{
The psychocognitive, biochemical, and electrophysiological effects of cerebral ischemia-reperfusion injury in patients undergoing carotid endarterectomy accompanied by cervical block
}

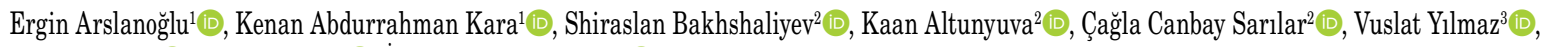
Görkem Şirin $^{3}$ (D) Nerses Bebek ${ }^{3}$ (D) İbrahim Ufuk Alpagut²

${ }^{1}$ Department of Pediatric Cardiovascular Surgery, Koşuyolu High Specialization Education and Research Hospital, Istanbul, Turkey

${ }^{2}$ Department of Cardiovascular Surgery, Istanbul University Istanbul Faculty of Medicine, Istanbul, Turkey

${ }^{3}$ Department of Neurology and Neuroscience, Istanbul University Istanbul Faculty of Medicine, Istanbul, Turkey

\begin{abstract}
Objectives: This study aims to examine perioperative changes and correlation of electroencephalography (EEG), neuropsychological test (NPT), and neurobiochemical markers in patients undergoing carotid endarterectomy.

Patients and methods: A total of 48 patients ( 28 males, 20 females; mean age: $68.4 \pm 7.7$ years; range, 43 to 84 years) who underwent carotid endarterectomy in our clinic between January 2017 and March 2019 were included in the study. Basic activity, slow-wave, and paroxysmal activities were evaluated using the pre- and postoperative EEG. The S100B protein (S100B), neuron-specific enolase (NSE), and glial fibrillary acidic protein (GFAP) values from biochemical markers were studied in all patients in the preoperative period, postoperative early period (Day 2), and postoperative late period. The NPT was examined preoperatively and at one month postoperatively.

Results: The EEG examination of 30 patients revealed a decrease in the slow-wave frequency and amplitude in the EEG of 10 patients without ischemia in the preoperative magnetic resonance imaging examination. The S100B and GFAP values decreased and the NSE values increased statistically significantly in the late postoperative period, although there was no statistically significant difference between the NSE, S100B, and GFAP values in the early postoperative period before and after carotid endarterectomy operation.

Conclusion: The positive effects of carotid endarterectomy on EEG in the patient group without ischemia can be interpreted as the electrophysiological evidence and that possible functional damage associated with carotid stenosis can be improved after endarterectomy. The values of biochemical markers decreased in the late postoperative period with the limitation of inflammation and NSE increased, which may be associated with neuronal regeneration.
\end{abstract}

Keywords: Carotid, carotid stenosis, electroencephalography, endarterectomy.

Carotid endarterectomy (CEA) is a successful treatment in patients with symptomatic or asymptomatic carotid artery critical stenosis. ${ }^{[1]}$ However, cerebral ischemia and related neurological damage may occur during CEA. ${ }^{[2]}$ A secondary inflammatory process may also occur due to cerebral ischemia-reperfusion injury after CEA and there may be changes in its associated or independent cognitive functions and electrophysiological results. Cerebral response to reperfusion of each patient is unique. ${ }^{[3]}$

During carotid surgery, electroencephalography (EEG) can be used to determine whether a perioperative carotid shunt is required. ${ }^{[4]}$ It is non-specific to ischemia, and anesthetics are affected by drugs, body temperature, hemodynamic changes.

Received: May 13, 2021 Accepted: June 13, 2021 Published online: July 26, 2021

Correspondence: Ergin Arslanoğlu, MD. Koşuyolu Yüksek İhtisas Eğitim ve Araştırma Hastanesi Çocuk Kalp Damar Cerrahisi Kliniği, 34865 Kartal, İstanbul, Türkiye. e-mail: erginarslanoglu@gmail.com 
The EEG alterations without permanent cell damage occur, when cerebral blood flow decreases from $18 \mathrm{~mL} / 100 \mathrm{~g} / \mathrm{min}$ to $10 \mathrm{~mL} / 100 \mathrm{~g} / \mathrm{min}$, although not all ischemia alterations result in cell death. ${ }^{[5]}$ These transient ischemic conditions cause changes in EEG, but permanent sequelae are uncommon in the postoperative period. ${ }^{[5]}$

The S100B protein (S100B), neuron-specific enolase (NSE), and glial fibrillary acidic protein (GFAP) values measured from peripheral blood are used as neurological inflammatory markers and are thought to be valuable in determining neuronal damage. ${ }^{[6]}$ Several studies have shown that these markers in astrocytes in the central nervous system are not correlated to the degree of neuronal damage, but they are known to increase after head trauma and heart surgery. ${ }^{[7,8]}$

Carotid stenosis has been associated with cognitive dysfunction. ${ }^{[9]}$ Hypoperfusion and cerebral atherosclerosis are the reasons. Approximately one-third of the patients undergoing the cognitive test after CEA have early fine cognitive regression, and this regression returns to normal after one week. ${ }^{[10]}$ Improvements in cognitive functions have been reported in some series. Microembolism that may occur during CEA and temporary ischemia during surgery may cause cognitive impairment. ${ }^{[1]}$

In the present study, we aimed to examine perioperative changes and correlation of EEG, neuropsychological test (NPT), cognitive functions, and neurobiochemical markers (S100B, NSE, GFAP) in patients undergoing CEA due to carotid stenosis.

\section{PATIENTS AND METHODS}

This prospective study was conducted at Istanbul University Istanbul Faculty of Medicine, Department of Cardiovascular Surgery between January 2017 and March 2019. A total of 48 patients (28 males, 20 females; mean age: $68.4 \pm 7.7$ years; range, 43 to 84 years) who had unilateral carotid stenosis and who attended to regular outpatient follow-up visit after CEA were included. All patients underwent preoperative magnetic resonance imaging (MRI). The CEA operations were performed under the cervical block. The need for a shunt was determined after performing a 3-min clamp test and, during surgery, patients' mental evaluations were performed by question-and-answer method, while motor functions were assessed based on contralateral limb movements. ${ }^{[12]}$ All patients were patched with a Dacron ${ }^{\circledR}$ patch after endarterectomy. All patients were followed in the intensive care unit for the first $24 \mathrm{~h}$ postoperatively. A written informed consent was obtained from each patient. The study protocol was approved by the Istanbul University, Istanbul Medical Faculty, Ethics Committee (Date: 29/05/2017; No: 567). The study was conducted in accordance with the principles of the Declaration of Helsinki.

\section{Electrophysiological evaluation}

Of 48 patients included in the study, 30 underwent EEG examination using the Neurofax EEG-1100 K ${ }^{\circledR}$ system (Nihon Kohden Corp., Tokyo, Japan) and Nihon Kohden ${ }^{\circledR}$ (Nihon Kohden Corp., Tokyo, Japan) brand device for approximately $20 \mathrm{~min}$ on postoperative Day 7 before and after CEA. The EEG recordings were performed through 18 channels with $\mathrm{Ag} / \mathrm{AgCl}$ electrodes placed on the scalp according to the international 10-20 system. The EEG data were digitized with a sampling rate of $100 \mathrm{~Hz}$ and recorded in a digital environment. Hyperventilation and photic stimulation activation methods were used for each patient for 5 min during EEG. The presence of basic activity and paroxysmal activity in the EEG examination was visually evaluated by an experienced clinical neurophysiologist. The dominant rhythm of the significant basic activity in the posterior regions was determined as frequency. The frequency of the slow waves was determined. Slow waves were classified according to their localization as unilateral right, unilateral left, and bilateral. Paroxysmal activities were considered intermittent rhythmic delta activity, periodic anomalies, focal or generalized sharp and spike-wave discharges. The frequency of slow waves and paroxysmal activities was rated infrequently, moderately, and frequently by the assessor. The EEG examinations could not be performed in 18 patients due to patient non-compliance.

\section{Neurobiochemical markers}

Serum samples were taken from all patients in the preoperative, postoperative early period (postoperative Day 2), and postoperative late period (postoperative Month 1). The GFAP (with Elabscience brand kit), S100B (with Diametra brand kit), and human NSE (hNSE) (with Diametra brand kit) tests were studied using the Cobas E411 (Roche Diagnostics, Mannheim, Germany) device with electrochemiluminescence method. The reading was performed at $450 \mathrm{~nm}$.

\section{Neuropsychological evaluation}

All patients diagnosed with carotid stenosis and operated underwent an NPT battery to measure 
Table 1. Pre- and postoperative comparisons of dominant basic activity frequency value in EEG

\begin{tabular}{|c|c|c|c|c|c|c|c|c|}
\hline \multirow[b]{2}{*}{ Frequency value } & \multicolumn{3}{|c|}{ Preoperative (n=30) } & \multicolumn{3}{|c|}{ Postoperative (n=30) } & \multirow{2}{*}{$\frac{\text { Difference }}{\text { Mean } \pm S D}$} & \multirow[b]{2}{*}{$p^{*}$} \\
\hline & Mean \pm SD & Median & Min-Max & Mean \pm SD & Median & Min-Max & & \\
\hline Hertz $(\mathrm{Hz})$ & $8.7 \pm 1.1$ & 8.5 & $5.5-10.5$ & $8.7 \pm 1.1$ & 8.5 & $5.5-10.5$ & $0.1 \pm 0.0$ & 0.102 \\
\hline
\end{tabular}

memory, attention, executive functions, and visual-spatial perception before and one month after surgery. A specialist psychologist in this battery performed the following tests: Öktem-Verbal Memory Processes Test (VMPT) to measure different dimensions of memory and Luria's Alternating Series Test, Digit Span Test, Categorical Verbal Fluency Tests, Stroop Test, Serial Sevens, Clock-Drawing Test, Luria's Alternating Series Test, and Benton Facial Recognition Test to measure visual-spatial perception. In addition, the sociodemographic information of the patients was collected through a form.

\section{Statistical analysis}

Statistical analysis was performed using the NCSS version 9 statistical software (NCSS LLC, Kaysville, UT, USA). Descriptive data were expressed in mean \pm standard deviation, median (min-max) or number and frequency. The Friedman test was used to compare quantitative data with three-point measurements in dependent groups without normal distribution, while the Wilcoxon signed-rank test was used to compare binary comparisons. The KruskalWallis test was used for multiple comparisons in independent groups, while the Mann-Whitney U test was used for binary comparisons. The Spearman correlation test was used to examine relationship between quantitative variables. A $p$ value of $<0.05$ was considered statistically significant.

\section{RESULTS}

Of the patients included in the study, 50\% $(n=24)$ were in the $57-70$ age range, $41.7 \%(n=20)$ were in the $71-84$ age range, and $8.3 \%(n=4)$ were in the 43-56 age range. Totally, $75 \%(n=36)$ of the patients were primary school graduates, $4.2 \%(n=2)$ were secondary school graduates, and $10.4 \%(n=5)$ were high school graduates, whereas $10.4 \%(n=5)$ were only literate. Of all patients, eight (16.6\%) needed a shunt. No additional complications were observed in the postoperative period.

\section{Electrophysiological evaluation results}

A total of $50 \%(n=15)$ of the patients had stenosis on the right side of the carotid artery, whereas 50\% $(\mathrm{n}=15)$ had stenosis on the left side. No statistically significant difference was found between the dominant frequency values of the basic activity in the pre- and postoperative periods $(\mathrm{p}=0.102$, Table 1$)$.

Of the patients who underwent EEG examination, 12 had no cortical-subcortical ischemia, while 18 had ischemia on the MRI examination. The slow-wave activity was detected in 19 patients in the postoperative period, whereas slow-wave activity was detected in 29 patients in the preoperative period. The slow-wave activity of 10 patients with slow-wave activity in the preoperative period disappeared in the postoperative period. The MRI examination findings were normal in all 10 patients with the improved EEG results. There was a statistically significant difference between the recovery status of the slow-wave in EEG examination according to whether a lesion was detected on MRI ( $p=0.001$, Table 2). The MRI results revealed that the recovery status of slow waves in the postoperative period was more frequent in the patient group without lesions, compared to the group without lesions. The worsening in the EEG examination was only observed in one patient and this patient had relevant MRI results. The change in frequency value and the slow-wave frequency value of the slow-wave before and after the operation was not statistically significant $(\mathrm{p}=0.219 ; \mathrm{p}=0.112$, respectively).

No statistically significant relationship was found between carotid stenosis sides of patients and slow-wave localizations in EEG in the preoperative period $(\mathrm{p}=0.160)$. The paroxysmal activity was observed in

\begin{tabular}{|c|c|c|c|c|c|}
\hline & \multicolumn{4}{|c|}{ Recovery status } & \multirow[b]{3}{*}{$p^{*}$} \\
\hline & \multicolumn{2}{|c|}{ No improvement } & \multicolumn{2}{|c|}{ Improvement } & \\
\hline & $\mathrm{n}$ & $\%$ & $\mathrm{n}$ & $\%$ & \\
\hline \multicolumn{6}{|c|}{ MRI lesion } \\
\hline No & 8 & 44.4 & 10 & 55.6 & \multirow{2}{*}{$0.001 \dagger$} \\
\hline Yes & 12 & 100 & - & - & \\
\hline
\end{tabular}

MRI: Magnetic resonance imaging; EEG: Electroencephalography; * $p<0.05$; † Fisher's exact test. 
Table 3. Comparison of GFAP value in blood over time

\begin{tabular}{|c|c|c|c|c|c|c|c|c|c|c|}
\hline \multirow[b]{2}{*}{ GFAP value } & \multicolumn{3}{|c|}{ Preoperative GFAP $(n=48)$} & \multicolumn{3}{|c|}{ Postoperative Day 2-GFAP (n=48) } & \multicolumn{3}{|c|}{ Postoperative Month 1-GFAP (n=48) } & \multirow[b]{2}{*}{$p^{\dagger}$} \\
\hline & Mean \pm SD & Median & Min-Max & Mean $\pm S D$ & Median & Min-Max & $\mathrm{Mean} \pm \mathrm{SD}$ & Median & Min-Max & \\
\hline$\mu \mathrm{g} / \mathrm{L}$ & $1.25 \pm 0.845$ & 1.25 & $0.08-3.25$ & $1.22 \pm 0.976$ & 1.17 & $0.92-3.32$ & $0.68 \pm 0.546$ & 0.34 & $0.11-1.51$ & $0.046^{*}$ \\
\hline
\end{tabular}

eight patients in the preoperative EEG, whereas it was detected in seven patients in the postoperative period. The spike-wave activity was observed in four patients and sharp wave activity was observed in three patients as paroxysmal activity. The presence of paroxysmal activity was not statistically significant in the postoperative period, compared to the preoperative period $(\mathrm{p}=0.999)$.

\section{Neuropsychological evaluation results}

The difference between the forward and backward attention span scores between the preoperative and postoperative period was found to be statistically significant $(p=0.003 ; p=0.002$ respectively). This difference was in favor of the postoperative period in positive ranks, when the rank totals of the different amounts were examined. The forward and backward attention span scores detected in the postoperative period were significantly higher, compared to the preoperative period.

Similarly, the Stroop test and VMPT short-term memory scores in the postoperative period were significantly higher, compared to the preoperative period ( $\mathrm{p}=0.040 ; \mathrm{p}=0.023$, respectively). However, there was no significant difference between the peri- and postoperative scores in terms of face recognition test score $(p=0.878 ; p>0.05)$, VMPT longterm memory score $(p=0.231 ; p>0.05)$, countdown score from 20 ( $p=0.143 ; p>0.05)$, counting days of the week score $(p=0.720 ; p>0.05)$, fluency score $(p=0.507$; $\mathrm{p}>0.05)$, A score $(\mathrm{p}=0.312 ; \mathrm{p}>0.05)$, counting animals score $(\mathrm{p}=0.842 ; \quad \mathrm{p}>0.05), \quad$ abstraction-similarity $(\mathrm{p}=0.317 ; \mathrm{p}>0.05)$, clock-drawing $(\mathrm{p}=0.08 ; \mathrm{p}>0.05)$, Luria's alternating series test score $(p=0.999 ; p>0.05)$, and copying shapes score $(\mathrm{p}=0.999 ; \mathrm{p}>0.05)$ among other tests.

\section{Neurobiochemical marker results}

The mean GFAP values in the blood were $1.25 \pm 0.845 \mu \mathrm{g} / \mathrm{L}$ in the preoperative period, $1.22 \pm 0.976 \mu \mathrm{g} / \mathrm{L}$ in the early postoperative period, and $0.68 \pm 0.546 \mu \mathrm{g} / \mathrm{L}$ in the late postoperative period. The change in GFAP values was found to be statistically significant according to the three different periods measured ( $p=0.046$, Table 3).

The mean $\mathrm{S} 100 \mathrm{~B}$ values in the blood were $0.07 \pm 0.008 \mu \mathrm{g} / \mathrm{L}$ in the preoperative period, $0.07 \pm 0.020 \mu \mathrm{g} / \mathrm{L}$ in the early postoperative period, and $0.68 \pm 0.546 \mu \mathrm{g} / \mathrm{L}$ in the late postoperative period. The change in $\mathrm{S} 100 \mathrm{~B}$ values was found to be statistically significant according to the three different periods measured ( $p=0.027$, Table 4).

The mean hNSE values in the blood were $0.42 \pm 0.316 \mu \mathrm{g} / \mathrm{L}$ in the preoperative period, $0.39 \pm 0.237 \mu \mathrm{g} / \mathrm{L}$ in the early postoperative period, and $0.70 \pm 0.442 \mu \mathrm{g} / \mathrm{L}$ in the late postoperative period. The change in hNSE values was found to be statistically significant according to the three different periods measured ( $p=0.006$, Table 5).

Table 4. Comparison of S100B value in blood over time

\begin{tabular}{|c|c|c|c|c|c|c|c|c|c|c|}
\hline \multirow[b]{2}{*}{ S100b value } & \multicolumn{3}{|c|}{ Preoperative-S100b (n=48) } & \multicolumn{3}{|c|}{ Postoperative-S100b (n=48) } & \multicolumn{3}{|c|}{ Late postoperative-S100b ( $\mathrm{n}=48$ ) } & \multirow[b]{2}{*}{$p_{\dagger}^{\dagger}$} \\
\hline & Mean $\pm S D$ & Median & Min-Max & Mean \pm SD & Median & Min-Max & Mean \pm SD & Median & Min-Max & \\
\hline$\mu \mathrm{g} / \mathrm{L}$ & $0.075 \pm 0.008$ & 0.07 & $0.05-0.10$ & $0.073 \pm 0.020$ & 0.07 & $0.05-0.16$ & $0.071 \pm 0.007$ & 0.07 & $0.05-0.09$ & $0.027^{*}$ \\
\hline
\end{tabular}

Table 5. Comparison of hNSE value in blood by time

\begin{tabular}{|c|c|c|c|c|c|c|c|c|c|c|}
\hline \multirow[b]{2}{*}{ hNSE value } & \multicolumn{3}{|c|}{ Preoperative-hNSE $(\mathrm{n}=48)$} & \multicolumn{3}{|c|}{ Postoperative-hNSE ( $\mathrm{n}=48$ ) } & \multicolumn{3}{|c|}{ Late postoperative-hNSE ( $\mathrm{n}=48$} & \multirow[b]{2}{*}{$p \dagger$} \\
\hline & Mean \pm SD & Median & Min-Max & Mean \pm SD & Median & Min-Max & Mean $\pm S D$ & Median & Min-Max & \\
\hline$\mu \mathrm{g} / \mathrm{L}$ & $0.42 \pm 0.316$ & 0.34 & $0.12-2.00$ & $0.39 \pm 0.237$ & 0.35 & 0.13-1.14 & $0.70 \pm 0.442$ & 0.56 & $0.15-1.26$ & $0.006^{*}$ \\
\hline
\end{tabular}




\begin{tabular}{|c|c|c|c|}
\hline & GFAP & S100b & hNSE \\
\hline \multicolumn{4}{|c|}{ Forward Attention Span Score } \\
\hline $\mathrm{r}$ & -0.104 & 0.155 & -0.040 \\
\hline$p$ & 0.481 & 0.294 & 0.786 \\
\hline \multicolumn{4}{|c|}{ Backward Attention Span Score } \\
\hline $\mathrm{r}$ & -0.073 & 0.182 & 0.193 \\
\hline$p$ & 0.620 & 0.216 & 0.379 \\
\hline \multicolumn{4}{|c|}{ Stroop Test Score } \\
\hline $\mathrm{r}$ & -0.160 & -0.181 & -0.119 \\
\hline$p$ & 0.277 & 0.219 & 0.422 \\
\hline \multicolumn{4}{|c|}{ VMPT Short-Term Memory Score } \\
\hline $\mathrm{r}$ & -0.102 & 0.158 & 0.186 \\
\hline$p$ & 0.161 & 0.284 & 0.206 \\
\hline
\end{tabular}

NPT: Neuropsychological test; GFAP: Glial fibrillary acidic protein; S100B: S100B protein; hNSE: Human neuron-specific enolase; Spearman's correlation test; $p<0.05$.

No statistically significant differences were found between S100B ( $\mathrm{p}=0.076)$, hNSE $(\mathrm{p}=0.104)$, and GFAP $(\mathrm{p}=0.229)$ values between patients with and without improvement in slow-wave activity in the postoperative period.

In addition, no significant difference was observed between the group whose EEG result improved and did not improve in the postoperative period in the forward and backward attention span tests $(p=0.206$ and $\mathrm{p}=0.414)$ and Stroop test $(\mathrm{p}=0.182)$ in the NPT examinations, and the VMPT short-term memory test showed a positive statistical significance between the two groups $(\mathrm{p}=0.013)$.

In the correlation analysis, no statistically significant relationship was found between forward and backward attention span scores, Stroop score, and VMPT short-term memory score, and GFAP, S100B, and hNSE values (Table 6).

\section{DISCUSSION}

Although EEG may provide information about cerebral perfusion in the postoperative follow-up of patients with carotid stenosis, studies on its use for this purpose are still limited. In the literature, there is no study comparing three of them, even though studies examining EEG, NPT, and neurobiochemical markers individually in patients undergoing CEA are available. In the present study, we investigated the correlation of these tests in the patients who underwent CEA in our clinic.

Chung et al. ${ }^{[1]}$ compared carotid stump pressure and EEG monitoring in their study with 50 patients who underwent CEA and reported that their combined use produced more significant results. ${ }^{[2]}$ Thirumala et al. ${ }^{[5]}$ also used EEG to evaluate the risk of stroke perioperatively and found significant EEG changes in stroke patients. In our study, we did not examine the basic activity and slow waves in 30 patients who underwent EEG in the pre- and postoperative early period (postoperative Day 7), and the EEG of 10 asymptomatic patients without ischemic lesions showed a decrease in slow-wave frequency and amplitude on preoperative MRI, demonstrating the contribution of CEA to the regulation of cerebral electrophysiology. The EEG examination was observed to be impaired in one of these 30 patients in the postoperative period. The reason for this can be asymptomatic plaque rupture, insomnia, or hunger. We observed no changes in the EEG examination of the remaining 19 patients. No ischemia was observed in preoperative MRI examinations of patients with improvement in slow-wave activity. It is an expected result that slow waves in EEG would not improve in the postoperative period, since slow waves in EEG in the presence of ischemia in MRI are associated with a lesion. These findings suggest that there is electrophysiological evidence of improvability of possible functional damage associated with carotid stenosis after CEA in the absence of ischemic lesion as assessed by MRI.

Zhang et al. ${ }^{[13]}$ obtained a decrease in the early period and statistically non-significant results in the late period considering the pre-, post-, and late-stage NPT results of patients with carotid stenosis in their study. Cognition was also shown to decrease with depressive disorders in the early period and Belov et al. ${ }^{[14]}$ obtained statistically non-significant results in their study including 120 patients. The patients' forward and backward attention span and short-term memory increased as a result of the NPT we performed in 48 patients in the pre- and postoperative first month in our study. The limitation of this result can be attributed to the fact that patients could learn the test; however, more precise results can be obtained by increasing the patient population in future studies.

The S100B, NSE, and GFAP values measured from peripheral blood are used as neurological inflammatory markers and are known to be valuable in the evaluation of neuronal damage. Nagy et al. ${ }^{[15]}$ examined the S100B value before and after carotid surgery and suggested that $\mathrm{S} 100 \mathrm{~b}$ decreased in the late period. The results of our study support this result. We also found an increase in NSE, whereas S100b and GFAP statistically significantly decreased in the 
late period. We believe that S100B and GFAP, which are the markers of inflammation, decrease with the limitation of inflammation, but NSE, which shows neuronal regeneration, increases with the increased permeability of the blood-brain barrier as a result of microembolism and complement activation.

In another study comparing stent and endarterectomy, Capoccia et al. ${ }^{[16]}$ used S100B and NSE to evaluate brain damage, although they found an increase within the first $24 \mathrm{~h}$ in the early period. The NSE and S100B values of the patients were not examined in the late period in this study. The NSE, S100B, and GFAP values were examined in the peripheral blood of 48 patients in the preoperative, postoperative early (Day 2), and postoperative late period (Month 1) in our study and a statistically significant difference was obtained only between late-period results and preoperative values. The significant decrease in the results in the late period was attributed to the self-limitation of inflammation. The S100B, NSE, and GFAP have been examined in many publications after cranial trauma and their values have been shown to increase with brain tissue damage. ${ }^{[8,17,18]}$ Considering the decline in the late period of these biochemical markers used in the context of early inflammation and damage, further comprehensive studies are needed to prove the benefit of endarterectomy and to use it in patient follow-up in the future.

It is known that contralateral stenosis may affect brain damage in patients with carotid stenosis and contralateral severe carotid stenosis is associated with an increased risk of death and stroke. ${ }^{[19]}$ In this study, we included only patients with unilateral severe stenosis.

Cognitive functions secondary to hyperfusion in the postoperative period decrease and intraoperative events may adversely affect postoperative cognition, despite the strict blood pressure and hemodynamic follow-up during the operation. ${ }^{[20]}$ A low level of increase was detected in short-term memory and attention span tests, despite the increased blood flow in the cognitive functions of the patients in the postoperative first month in our study. The patients' highest education level was high school or lower in this study, which limits the generalizability of the results.

Furthermore, Wardlaw et al. ${ }^{[21]}$ suggested that there was a relationship between lacunar infarcts and progressive cognitive impairment or dementia in the pathogenesis of impaired permeability in the blood-brain barrier. In our study, we found no statistically significant correlation postoperative biochemical markers and NPT. An NPT that reflects frontal region activity such as the Stroop test may mislead us in the presence of an infarction affecting other parts of the brain, and we believe that these harmless, easily accessible cognitive tests can be used in the prognosis of cerebral inflammation and repair processes, although learning the test is known to be limiting for Stroop test, whether these tests are used alone in the follow-up of patients undergoing CEA or not. Additionally, some improvements in the neurocognitive functions were observed after revascularization in patients with impaired cognition in previous studies. ${ }^{[22,23]}$ Attention span and Stroop tests were slightly increased in our study.

Nonetheless, there are some limitations to this study. Biochemical markers are diagnostic methods that can be used in the follow-up of EEG and NPT carotid patients, although the correlation with each other is statistically non-significant. The fact that biochemical markers are directly related to inflammation and labelled from ischemic lesions and events and the different inflammatory responses of each patient make it difficult to use biochemical markers in daily practice. Similarly, it is challenging to make clear decisions, as EEG is affected by noise, hunger, or insomnia, and is a very sensitive method during administration. This makes it difficult for EEG to be a routine diagnostic and follow-up tool in carotid patients. The fact that NPT did not increase significantly in the postoperative first month can be attributed to additional age-related diseases and past neurological events in patients. The low level of socio-cultural education of our patient group can be also deemed as a limitation.

In conclusion, the EEG is a valuable tool in the peri- and postoperative follow-up of patients with carotid stenosis. In our study, the $\mathrm{S} 100 \mathrm{~b}$ and GFAP statistically significantly decreased and hNSE increased in the late postoperative period, whereas there was no change in the S100B, GFAP, and NSE values of neurobiochemical markers in the early period. This result suggests that biochemical markers are more valuable in the subacute and chronic processes. A slight increase in attention short-term memory was also detected in the postoperative first month as a result of NPT performed in patients undergoing CEA. Carotid endarterectomy is a favorable treatment option that can provide neurophysiological and cognitive improvement in patients with carotid stenosis. 


\section{Declaration of conflicting interests}

The authors declared no conflicts of interest with respect to the authorship and/or publication of this article.

\section{Funding}

This study was supported by the Istanbul University Scientific Research Projects Coordination Unit with the Project No. 26406.

\section{REFERENCES}

1. Chang R, Reddy RP, Sudadi S, Balzer J, Crammond DJ, Anetakis K, et al. Diagnostic accuracy of various EEG changes during carotid endarterectomy to detect 30-day perioperative stroke: A systematic review. Clin Neurophysiol 2020;131:1508-16.

2. Di Legge S, Di Piero V, Di Stani F, Perna R, Gattuso R, Reale MG, et al. Carotid endarterectomy and gliofibrillar S100b protein release. Neurol Sci 2003;24:351-6.

3. Witt K, Börsch K, Daniels C, Walluscheck K, Alfke $\mathrm{K}$, Jansen $\mathrm{O}$, et al. Neuropsychological consequences of endarterectomy and endovascular angioplasty with stent placement for treatment of symptomatic carotid stenosis: A prospective randomised study. J Neurol 2007;254:1524-32.

4. Cho I, Smullens SN, Streletz LJ, Fariello RG. The value of intraoperative EEG monitoring during carotid endarterectomy. Ann Neurol 1986;20:508-12.

5. Thirumala PD, Thiagarajan K, Gedela S, Crammond DJ, Balzer JR. Diagnostic accuracy of EEG changes during carotid endarterectomy in predicting perioperative strokes. J Clin Neurosci 2016;25:1-9.

6. Connolly ES Jr, Winfree CJ, Rampersad A, Sharma R, Mack WJ, Mocco J, et al. Serum S100B protein levels are correlated with subclinical neurocognitive declines after carotid endarterectomy. Neurosurgery 2001;49:1076-82.

7. Herrmann M, Ebert AD, Galazky I, Wunderlich MT, Kunz WS, Huth C. Neurobehavioral outcome prediction after cardiac surgery: Role of neurobiochemical markers of damage to neuronal and glial brain tissue. Stroke 2000;31:645-50.

8. Herrmann M, Curio N, Jost S, Grubich C, Ebert AD, Fork ML, et al. Release of biochemical markers of damage to neuronal and glial brain tissue is associated with short and long term neuropsychological outcome after traumatic brain injury. J Neurol Neurosurg Psychiatry 2001;70:95-100.

9. Heyer EJ, Adams DC, Solomon RA, Todd GJ, Quest DO, McMahon DJ, et al. Neuropsychometric changes in patients after carotid endarterectomy. Stroke 1998;29:1110-5.

10. Heyer EJ, Sharma R, Rampersad A, Winfree CJ, Mack WJ, Solomon RA, et al. A controlled prospective study of neuropsychological dysfunction following carotid endarterectomy. Arch Neurol 2002;59:217-22.

11. Valenti P, Ortelli P, Zanon A, Schiff S, Montagnese S, Avruscio G, et al. Psychometric and EEG changes after carotid endarterectomy. Metab Brain Dis 2015;30:99-105.

12. Kavrut Ozturk N, Kavakli AS, Sagdic K, Inanoglu K, Umot Ayoglu R. A randomized controlled trial examining the effect of the addition of the mandibular block to cervical plexus block for carotid endarterectomy. J Cardiothorac Vasc Anesth 2018;32:877-82.

13. Zhang HP, Ma XD, Chen LF, Yang Y, Xu BN, Zhou DB. Cognitive function after carotid endarterectomy: Early decline and later recovery. Turk Neurosurg 2016;26:833-9.

14. Belov YV, Medvedeva LA, Zagorulko OI, Charchyan ER, Drakina OV. Early and long-term cognitive disorders after carotid endarterectomy. Khirurgiia (Mosk) 2018;12:5-12.

15. Nagy B, Woth G, Mérei Á, Nagy L, Lantos J, Menyhei G, et al. Perioperative time course of matrix metalloproteinase-9 (MMP-9), its tissue inhibitor TIMP-1 \& S100B protein in carotid surgery. Indian J Med Res 2016;143:220-6.

16. Capoccia L, Speziale F, Gazzetti M, Mariani P, Rizzo A, Mansour W, et al. Comparative study on carotid revascularization (endarterectomy vs stenting) using markers of cellular brain injury, neuropsychometric tests, and diffusion-weighted magnetic resonance imaging. J Vasc Surg 2010;51:584-91.

17. Oris C, Pereira B, Durif J, Simon-Pimmel J, Castellani C, Manzano S, et al. The biomarker S100B and mild traumatic brain injury: A meta-analysis. Pediatrics 2018;141:e20180037.

18. Martens P, Raabe A, Johnsson P. Serum S-100 and neuronspecific enolase for prediction of regaining consciousness after global cerebral ischemia. Stroke 1998;29:2363-6.

19. Ghogawala Z, Amin-Hanjani S, Curran J, Ciarleglio M, Berenstein A, Stabile L, et al. The effect of carotid endarterectomy on cerebral blood flow and cognitive function. J Stroke Cerebrovasc Dis 2013;22:1029-37.

20. Erdolu B, Engin M. The effect of mean platelet volume-tolymphocyte ratio on symptom onset in patients with carotid artery stenosis. Turk J Vasc Surg 2021;30:7-12.

21. Wardlaw JM, Sandercock PA, Dennis MS, Starr J. Is breakdown of the blood-brain barrier responsible for lacunar stroke, leukoaraiosis, and dementia? Stroke 2003;34:806-12.

22. Siddiqui AH, Hopkins LN. Asymptomatic carotid stenosis: The not-so-silent disease: Changing perspectives from thromboembolism to cognition. J Am Coll Cardiol 2013;61:2510-3.

23. Huang CC, Chen YH, Lin MS, Lin CH, Li HY, Chiu MJ, et al. Association of the recovery of objective abnormal cerebral perfusion with neurocognitive improvement after carotid revascularization. J Am Coll Cardiol 2013;61:2503-9. 